\title{
Transparenz allein reicht bei Interessenkonflikten nicht aus - Kontra
}

\author{
Transparency Alone is not Sufficient for the Management of Conflicts \\ of Interest - Contra
}

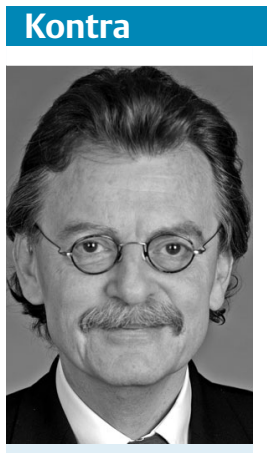

Jürgen Fritze
Als mich die Herausgeber der Psychiatrischen Praxis eingeladen haben, zu dieser Debatte beizutragen, habe ich mich als ungeeignet erklärt, weil ich mir zugutehalte, Anfang des Jahrtausends an der Einführung der Pflicht zur Offenlegung von Interessenkonflikten (COI) bei der Deutschen Gesellschaft für Psychiatrie und Psychotherapie, Psychosomatik und Nervenheilkunde e.V. (DGPPN) mitgewirkt zu haben. Trotzdem...

Transparenz der COI ist unverzichtbar, nicht - wie üblich - schamhaft klein gedruckt am Ende einer Publikation versteckt, sondern am Anfang. In den letzten 2 Jahren habe ich Honorare für Beratertätigkeiten von Janssen, Lundbeck, Lilly, Pfizer, Roche, Novartis, St. Jude Medical, $3 \mathrm{M}$, Sanvartis und dem Verband der privaten Krankenversicherung e.V. erhalten. Ich behandle seit 1996 keine Patienten. Ich lebe von Beratertätigkeiten. $\mathrm{Zu}$ meiner Mission gehört aber weiterhin - auch nach Aufgabe meines Amtes im Vorstand der DGPPN (2010) - die Sache der psychisch Kranken. Offensichtlich leide ich also unter stark divergierenden Interessen.

Zahlreiche Untersuchungen, auch im Kontext der psychiatrischen Forschung (z.B. [1]), haben offenbart, dass wissenschaftliche Ergebnisse verzerrt publiziert wurden und dabei ein Zusammenhang mit Interessenkonflikten naheliegt. Das Verhalten des forschenden Arztes muss aber ausschließlich von wissenschaftlicher Integrität bestimmt sein. Verzerrung infolge COI ist letztlich wissenschaftlicher Betrug, der riesige Schäden auf vielfältigen Ebenen bewirkt, bis hin zur Schädigung großer Patientengruppen. Das diagnostische und therapeutische Handeln des Arztes muss ausschließlich vom Bedarf des Kran- ken bestimmt sein, also frei von jedweden anderen Interessen bleiben, denn anderenfalls wird der Patient betrogen oder gar geschädigt.

Die Offenlegung von COI kann aber kein Selbstzweck sein, sondern ist Hilfsmittel, um die Aufmerksamkeit für mögliche interessengeleitete Verzerrungen in Forschung und Krankenbehandlung zu schärfen. Eigentliches Ziel muss sein, solche Verzerrungen zu verhindern. Ob uns die derzeitige Praxis der Offenlegung von COI diesen Zielen nähergebracht hat, ist unbekannt und bedarf also der Forschung. Inwiefern die optionale (Deutschland) oder obligate (USA) Offenlegung der Geldflüsse (oder das „Aussperren“ von Experten in der Arzneimittelkommission der deutschen Ärzteschaft - AkdÄ) das Erreichen dieser Ziele befördert, liegt im Dunkeln.

Dabei erscheint zumindest verwunderlich, dass man sich auf finanzielle COI, und hier insbesondere Zuwendungen der pharmazeutischen Industrie, eingeschossen hat und dabei ausschließlich den Arzt als Wissenschaftler (und komischerweise kaum andere Wissenschaftler) im Fokus hat. Angesichts dieses Mangels plant die Bundesregierung gemäß Koalitionsvertrag: „Bestechung und Bestechlichkeit im Gesundheitswesen wollen wir unter Strafe stellen." Auch das aber greift zu kurz, denn Bestechung und Bestechlichkeit stellen nur Extremformen interessengeleiteten Verhaltens dar. Die Zeiten, in denen dem Arzt (und anderen) zum Beispiel Kongressreisen von Dritten finanziert werden (also ohne Gegenleistung), sollten vorüber sein. Aber dem Arzt (u.a. Wissenschaftlern) muss möglich bleiben, ohne Stigmatisierung mit der Industrie zu kooperieren und für der Industrie geleistete Dienste (z.B. Beratung) angemessene Vergütungen - wie für jede andere Arbeit auch - zu erhalten.

Die Bürgerinnen und Bürger scheinen sich damit abgefunden oder gar angefreundet zu haben, auf einen Homo oeconomicus reduziert (und von der Politik mit „Anrei- zen“ dressiert) zu werden. Nichtfinanzielle COI werden bisher weitgehend ignoriert, jedenfalls nicht transparent gemacht. Immerhin wird debattiert, inwiefern Fallzahlsteigerungen im DRG-System „vom Arzt verinnerlichten“ ökonomischen Interessen des Krankenhauses geschuldet sein könnten (z. B. [2]).

Mario Maj [3], Präsident (2008-2011) der World Psychiatric Association (WPA), nennt daneben u.a. persönliche Anerkennung, Reputation, Karriereförderung, Medienaufmerksamkeit, Begünstigung von Verwandten, Freunden, Kollegen, Loyalität zu einer „Schule“ oder Lehrmeinung (z.B. für oder gegen Psychopharmaka), politisches Bekenntnis. Für den psychotherapiebedürftigen Patienten dürfte relevant sein, welcher „PsychotherapieSchule“ sich der mehr oder weniger zufällig kontaktierte Psychotherapeut „verpflichtet“ fühlt; welche Evidenz in seinem Einzelfall dafür oder dagegen spricht, dürfte sich seinem Urteil entziehen, zumal es nicht am Praxisschild zu stehen pflegt. Mario Maj zitiert den Review von Luborsky et al. [4], wonach die Loyalität zur „Psychotherapie-Schule“ 69\% der Effektstärke der Outcomes in Studien mit Vergleich dreier psychotherapeutischer Verfahren erklärte.

Die Agency for Healthcare Research and Quality (AHRQ) hat 2013 einen Konsensusbericht [5] vorgelegt, wie mit nichtfinanziellen COI im Rahmen systematischer Reviews (wohl auch darüber hinaus) umzugehen sei. Hier wird u.a. die explizite Offenlegung empfohlen - und Forschung zu deren Wirksamkeit.

Erübrigt sich eigentlich die Zusammenfassung: Mögliche COI des Arztes und ärztlichen Wissenschaftlers müssen nicht nur der scientific community, sondern auch dem einzelnen Patienten transparent werden. Diese Transparenz darf aber nicht vom Ziel ablenken: jedenfalls ärztliches Handeln - wissenschaftlich und therapeutisch - muss frei von konkurrierenden Interessen sein. 


\section{Interessenkonflikt}

$\nabla$

Jürgen Fritze erhielt Honorar für Beratertätigkeiten von Janssen, Lundbeck, Lilly, Pfizer, BMS, AstraZeneca, Roche, Novartis, St. Jude Medical, 3M, Sanvartis und dem Verband der privaten Krankenversicherung e.V..

\section{Literatur}

1 Turner EH, Matthews AM, Linardatos E et al. Selective publication of antidepressant trials and its influence on apparent efficacy. $\mathrm{N}$ Engl J Med 2008; 358: 252 - 260

2 Flintrop J. Krankenhäuser zwischen Medizin und Ökonomie: Die Suche nach dem richtigen Maß. Dtsch Arztebl 2014; 111: A-1929/ B-1649/C-1581

$3 \mathrm{Maj}$ M. Non-financial conflicts of interests in psychiatric research and practice. BJP 2008; 193: $91-92$
4 Luborsky L, Diguer L, Seligman DA et al. The researcher's own therapy allegiance: a "wild card" in comparisons of treatment efficacy. Clin Psychol Sci Prac 1999; 6: 95-106

5 Viswanathan M, Carey TS, Belinson SE et al. Identifying and Managing Nonfinancial Conflicts of Interest for Systematic Reviews. Methods Research Report. (Prepared by the RTI International-University of North Carolina at Chapel Hill Evidence-based Practice Center under Contract No. 290-200710056-I.) AHRQ Publication No.13-EHC085EF. Rockville, MD: Agency for Healthcare Research and Quality; 05.2013: www.effec tivehealthcare.ahrq.gov/reports/final.cfm

Sie haben eine eigene Meinung zu diesem

Thema? Dann schreiben Sie uns an:

psychiat-praxis@thieme.de!

\section{Korrespondenzadresse}

\section{Prof. Dr. med. Jürgen Fritze}

Arzt für Neurologie \& Psychiatrie, apl. Professor für Psychiatrie, Universität Frankfurt am Main Asternweg 65

50259 Pulheim

juergen.fritze@dgn.de

\section{Bibliografie}

Dol http://dx.doi.org/

10.1055/s-0034-1387518

Psychiat Prax 2015; 42: 14

(c) Georg Thieme Verlag KG

Stuttgart · New York

ISSN 0303-4259 